\section{REVISTA}

Actualidades Investigativas en Educación

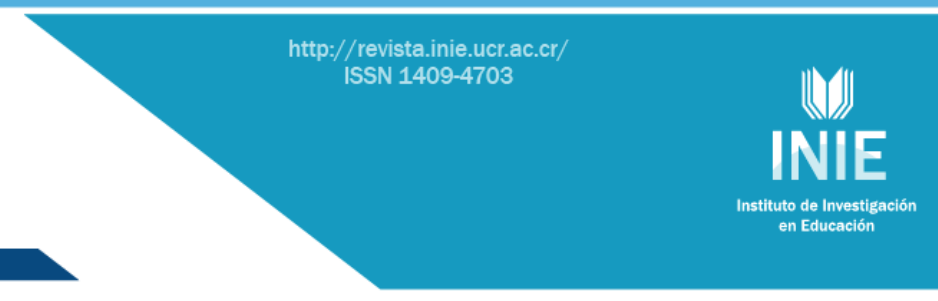

\title{
ACCESIBILIDAD AL PROCESO EDUCATIVO EN EL ENTORNO UNIVERSITARIO
}

ACCESSIBILITY OF THE EDUCATIONAL PROCESS IN THE UNIVERSITY ENVIRONMENT

\author{
Volumen 16, Número 1 \\ Enero - Abril \\ pp. 1-17
}

Este número se publicó el $1^{\circ}$ de enero de 2016

DOI: http://dx.doi.org/10.15517/aie.v16i1.21920

Martha Gross Martínez

Revista indizada en REDALYC, $\underline{\text { SCIELO }}$

Revista distribuida en las bases de datos:

LATINDEX, DOAJ, E-REVIST@S, IRESIE, CLASE, DIALNET, SHERPA/ROMEO, QUALIS,

Revista registrada en los directorios:

ULRICH'S, REDIE, RINACE, OEI, MAESTROTECA, PREAL, CLACSO

Los contenidos de este artículo están bajo una licencia Creative Commons

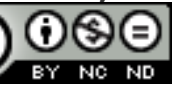




\title{
ACCESIBILIDAD AL PROCESO EDUCATIVO EN EL ENTORNO UNIVERSITARIO \\ ACCESSIBILITY OF THE EDUCATIONAL PROCESS IN THE UNIVERSITY ENVIRONMENT
}

\section{Martha Gross Martínez ${ }^{1}$}

Resumen: Este artículo presenta los hallazgos de la investigación Accesibilidad en la permanencia de estudiantes con necesidades educativas asociadas o no a discapacidad, en una universidad pública de Costa Rica (2012-2014). El objetivo de dicho estudio consistió en analizar la accesibilidad desde la percepción estudiantil sobre las acciones, apoyos y servicios dentro de la vida universitaria, a partir de las diferentes dimensiones de la accesibilidad (espacio físico, información y proceso educativo) y con ello aportar propuestas que permitan y dinamicen la inclusión en este nivel educativo. Este estudio se adscribe a la investigación cualitativa. La población participante se seleccionó considerando las necesidades de accesibilidad en el entorno universitario. Se expone de manera particular el análisis de los resultados sobre accesibilidad al proceso educativo en este contexto. Se realizó una revisión bibliográfica que permitiera la reflexión sobre el objeto de estudio, además, se llevó a cabo una encuesta en línea al estudiantado y la realización de grupos focales para la recolección de datos. Finalmente, se pone de manifiesto los logros y carencias que existen en materia de accesibilidad en el ámbito educativo, puntualizando aspectos vinculantes con el derecho a la educación y el compromiso institucional, ante los desafíos que esta población enfrenta en el quehacer de la vida estudiantil.

Palabras clave: INCLUSIÓN EDUCATIVA, EDUCACIÓN SUPERIOR, IGUALDAD DE OPORTUNIDADES, NECESIDADES DE EDUCACIÓN, DISCAPACIDAD, COSTA RICA

\begin{abstract}
This article presents the findings obtained in the research Accessibility in the Permanence of Students with Educational Needs Related, or not, to a Disability at a public university in Costa Rica (2012-2014). The objective of this study was to analyze accessibility from the students' perception regarding the actions, supports, and services of the university life, from the different accessibility dimensions (physical space, information, and educational process) and use it to offer proposals that can help and boost the educational inclusion in the higher education. This study is performed as a qualitative research, and the sample of the participating population was selected taking into consideration the needs for accessibility of the student body. The analysis of the results regarding accessibility in the educational process is presented in a particular manner. The research process was done with a review of the bibliographic information that permitted the reflection of the object of study; furthermore, an online survey was conducted with the students and focal groups were used to collect data. Finally, the accomplishments and deficiencies that exist in the topic of accessibility in the educational environment are clearly stated, specifying aspects related to the right of education and the institutional compromise to the challenges this population faces in its everyday student life.
\end{abstract}

Key words: ACCESS TO EDUCATION, HIGHER EDUCATION, EQUALITY OF OPPORTUNITIES, EDUCATIONAL NEEDS, DISABILITIES, COSTA RICA

\footnotetext{
${ }_{1}^{1}$ Docente de la carrera de Educación Especial, Universidad de Costa Rica. Investigadora del Instituto de Investigación en Educación (INIE). Máster en Psicopedagogía y Licenciada en Educación Especial.
}

Dirección electrónica: martha.gross@ucr.ac.cr

Artículo recibido: 27 de abril, 2015

Enviado a corrección: 4 de agosto, 2015

Aprobado: 9 de noviembre, 2015 


\section{Introducción}

La diversidad estudiantil en la educación superior conlleva el desafío de la eliminación de barreras, prejuicios y discriminación, lo que contribuye a la construcción de un entorno universitario en equidad e igualdad de oportunidades. Castellana y Sala (2005) mencionan que la creación de aulas inclusivas es un tema que no ha sido tratado de manera amplia en el campo de los estudios universitarios. De ahí el interés de profundizar sobre la inclusión y la accesibilidad en los procesos de formación en la educación superior, conceptualizando las dimensiones que la conforman y reconociendo aquellos elementos que sustentan y favorecen el derecho a la educación.

A partir de la investigación "Accesibilidad en la permanencia de estudiantes con necesidades educativas asociadas o no a discapacidad: Sede Rodrigo Facio" (Gross y Stiller, 2014), del Instituto de Investigación en Educación (INIE), de la Universidad de Costa Rica, se rescató la relevancia de las acciones, apoyos y servicios dentro de la vida estudiantil universitaria, a través de la sistematización de la percepción estudiantil sobre accesibilidad y con ello aportar propuestas que potencien y dinamicen la inclusión en la educación superior.

La inclusión de la población estudiantil con necesidades educativas asociadas o no a discapacidad es un proceso que plantea una proyección continua de metas y procedimientos, superando obstáculos para transformar el sistema educativo y dar respuesta a la diversidad de las necesidades del estudiantado; en todas las dimensiones de accesibilidad del quehacer universitario: educativo, informativo y espacio físico.

La conceptualización de necesidades educativas asociadas o no a discapacidad se acuña desde la experiencia de la Universidad de Costa Rica, concretamente desde el Centro de Asesoría y Servicios a Estudiantes con Discapacidad, el cual proporciona apoyos y servicios a estudiantes con una diversidad de necesidades educativas asociadas o no a una condición de discapacidad

Desde esa perspectiva, para efectos de la investigación, el concepto de accesibilidad se consideró como parte vital de la inclusión educativa universitaria, en cuanto a la provisión de recursos, apoyos, servicios y lineamientos que garanticen y fortalezcan el acceso pleno a todos los aspectos de la vida universitaria. En este sentido, se rescata la Convención de los Derechos de las Personas con Discapacidad (Asamblea Legislativa, 2008), Artículo 9, la cual fundamenta de manera contundente el acceso en igualdad de condiciones a: la educación, el entorno físico, la información y las comunicaciones, incluidos los sistemas y las tecnologías 
de la información y las comunicaciones, así como la identificación y eliminación de obstáculos y barreras de acceso.

De esta manera, la Universidad de Costa Rica se reconoce e identifica como una institución educativa precursora en temas y procesos que responden a una formación integral y de calidad que da respuesta a la comunidad estudiantil. Asimismo, la accesibilidad se ha convertido en un eje conceptual de importancia, presente en las políticas y acciones universitarias, lo cual ha generado transformaciones en la manera de planificar y actuar con respecto a la diversidad existente.

\section{Antecedentes del proceso investigativo}

Para una educación inclusiva es importante conceptuar la diversidad como una oportunidad para el aprendizaje y el desarrollo personal-social. Esto involucra la gestión de políticas educativas y marcos legales que sustentan la cobertura, provisión de apoyos, servicios y recursos en beneficio de la calidad de la educación y el compromiso con el cambio.

Algunos aspectos que todavía requieren ser fortalecidos son los procesos de concientización, apoyos y servicios dentro de la comunidad universitaria, contemplando un trabajo directo con todas y todos los participantes del quehacer institucional. Esto implica la divulgación de información y estrategias con metas asertivas que permitan no solo tener el conocimiento necesario, sino los instrumentos que facilitan y conduzcan a una universidad inclusiva a través de los diferentes actores sociales que la conforman.

La Universidad de Costa Rica ha contado con instancias que, de una u otra forma, fortalecen y promueven la accesibilidad universitaria como son: el Centro de Asesoría y Atención al Estudiante con Discapacidad (CASED), la Escuela de Orientación y Educación Especial, el Sistema Integrado de Bibliotecas, Documentación e Información (SIBDI), la Comisión Institucional en Materia de Discapacidad (CIMAD), los cuales han marcado una diferencia sustantiva en la calidad y apoyo de servicios, acciones y estrategias en procura de una universidad accesible.

De igual forma, se reconoce como la Universidad de Costa Rica ha hecho esfuerzos para proveer apoyos para el acceso a la educación al estudiantado con discapacidad, previo a la existencia de la Ley 7600 de Igualdad de Oportunidades (1996). Sin embargo, este marco legal garantiza el fortalecimiento y sistematización de las acciones que se emprenden en cada uno de estos centros de educación superior (Stupp, 2005), con lo cual la educación 
universitaria se convierte en una oportunidad para grupos tradicionalmente segregados, en ese sentido Chiroleu (2013) expresa:

En un contexto de creciente desigualdad y segmentación social la educación superior se constituye para diversos sectores sociales en una herramienta privilegiada para pugnar por una mejor inserción en el espectro social, a la vez que simultáneamente parece poder garantizar niveles de cohesión e integración en una sociedad profundamente fragmentada. (p. 7)

Siguiendo esa misma línea conceptual, se rescatan los acuerdos asumidos en la Convención de los Derechos de las Personas con Discapacidad, con un reto claro sobre el derecho de una educación para todos, Artículo 24, inciso 5, en el cual se exhorta sobre el acceso a la educación superior y la formación profesional sin discriminación y en igualdad de condiciones, esto constituye un reto para el sistema educativo en general.

Como parte de la aproximación teórica a la temática de la accesibilidad en el entorno universitario, también cabe destacar el estudio de Ramírez (2010): en éste abordan las acciones que la Universidad de Costa Rica ha llevado a cabo en materia de accesibilidad para los y las estudiantes en condición de discapacidad, desde una perspectiva de género. La investigadora aporta como resultado significativo el análisis de una coyuntura de cambio a lo interno de la universidad y muestra la carencia en cuanto a la forma más acertada para articular las acciones encaminadas hacia el tema de discapacidad y accesibilidad. De esta forma, señala que es importante explorar a través de otros estudios los diferentes contextos de la sociedad como un todo, abarcando distintas realidades con el fin de rescatar las similitudes y diferencias, que permiten la construcción de una sociedad que fortalece la equidad bajo el enfoque de derechos humanos.

De igual forma, es importante rescatar que en cuanto a la conceptualización de inclusión educativa, es fundamental el concepto de diversidad sustentado en estudios y aportes de autores como Arnaiz (2003), Sánchez y Pulido (2007), Sánchez (2011), los cuales defienden el derecho a una educación para todos, con una participación en equidad.

Así, con la intención de sistematizar la experiencia de los procesos vivenciados por el estudiantado con necesidades educativas asociadas o no a discapacidad, en el entorno universitario, se propuso este estudio para fundamentar, conceptualizar y evidenciar los logros y carencias en la educación superior en materia de accesibilidad. 


\section{Descripción metodológica}

Esta investigación se enmarca desde un enfoque cualitativo y surge de las inquietudes generadas por la población estudiantil con necesidades educativas asociadas o no a discapacidad, adscrita al Artículo 37 del Reglamento de Régimen Académico Estudiantil, de la Universidad de Costa Rica.

En la etapa inicial del proceso investigativo se propuso realizar una revisión de información bibliográfica que permitiera la reflexión sobre el objeto de estudio. Los documentos encontrados en las bases de datos consistieron en investigaciones, artículos científicos, trabajos finales de graduación, ensayos, ponencias, guías y protocolos sobre accesibilidad en ámbitos universitarios, tanto en el contexto nacional como internacional. La mayoría de los documentos recopilados provienen de Latinoamérica, España y en menor cantidad del Reino Unido y Estados Unidos. La búsqueda bibliográfica en la temática de accesibilidad, visualiza patrones de transformaciones conceptuales, que ido evolucionando con la creación de nuevos acuerdos internacionales y legislaciones nacionales; así como el desarrollo de acciones y medidas que se modifican a partir de procesos particulares en la sociedad. Al respecto Chiny, Salas y Vargas (2008) señalan que:

Considerando las profundas transformaciones ocurridas en torno a la atención de las personas con discapacidad en el ámbito universitario, se requiere de la reflexión constante y de un diagnóstico social, institucional y de aula que permitan acercarnos y conocer al otro para una mayor comprensión de sus circunstancias y las del entorno. (p. 78)

La investigación se llevó a cabo con la participación de una muestra conformada por 125 estudiantes con necesidades educativas asociada o no a discapacidad, adscritas al artículo 37 del Reglamento de régimen académico estudiantil. Para la recolección de datos se les invitó a participar en una encuesta en línea y la información obtenida se fue almacenando en el sistema conforme las personas iban contestándola. Del total de los 125 encuestados dieron respuesta 106 estudiantes, representando un 95\% de la muestra seleccionada. Esto se logró a través de recordatorios desde el portal del INIE lo cual permitió tener una participación amplia de la población muestra.

Otras de las técnicas utilizadas fueron los grupos focales, los cuales permitieron que cada participante comentara sobre su vivencia en el entorno universitario desde las tres dimensiones de accesibilidad propuestas para la investigación: proceso educativo, 
información y espacio físico. En el intercambio se expresaron opiniones de las y los estudiantes en relación con los aspectos que les han permitido avanzar en su vida universitaria, así como aquellas experiencias no tan positivas que han representado barreras relevantes en el contexto de su proyecto educativo.

Finalmente, se consideró la revisión de documentos sobre las adecuaciones y apoyos brindados a la población estudiantil adscrita al Artículo 37 al Reglamente de Régimen Académico Estudiantil, obteniendo datos que permitieron obtener un panorama amplio de la dimensión de accesibilidad al proceso educativo.

\section{Resultados: accesibilidad al proceso educativo}

En este proceso investigativo se establecieron categorías conceptuales designadas como dimensiones de la accesibilidad, las cuales se constituyeron en un sustento teórico fundamental para la clasificación, abordaje y análisis de la información recolectada. Estas comprenden elementos primordiales para la inclusión educativa en el ámbito universitario que garantizan tanto el acceso como la participación de la población estudiantil con necesidades educativas asociadas o no a discapacidad. Para efectos de este artículo, se expone de manera particular el análisis de los resultados sobre la dimensión de la accesibilidad al proceso educativo, la cual se conceptualizó como la disposición de los recursos necesarios, para que el estudiante participe en igualdad de condiciones en el proceso de enseñanza-aprendizaje, en un entorno inclusivo. Así, Espinosa, Gómez y Cañedo (2012) señalan:

La Universidad inclusiva debe ser aquel lugar donde todos son aceptados y apoyados por todos para que tengan cubiertas sus necesidades educativas, la idea debe ser replanteada de manera que se interiorice lo necesidad del tránsito de una universidad basada en la evaluación de la competencia docente o la asimilación de las competencias generales y específicas en los estudiantes a una universidad donde se evalúe adicionalmente la cooperación entre pares para favorecer la inclusión (estudiante - estudiante, docente - docente, docente - estudiante, etc.). (p. 3)

Salinas, Lissi, Medrano, Zuzulich y Hojas (2013) rescatan diferentes estudios y autores que señalan los factores que inciden en el acceso a la educación en el entorno universitario, así mencionan aquellos que plantean las barreras en los procesos de enseñanzaaprendizaje los cuales indican principalmente fallas en la accesibilidad a los recursos 
utilizados en dichos espacios, como son las actividades de aula y evaluativas; por otra parte señalan estudios que evidencian dificultades en la accesibilidad a material específico. Asimismo, se rescatan estudios que evidencian aportes significativos sobre el desconocimiento de los docentes universitarios y su falta de formación en temas como la discapacidad y metodologías inclusivas (Castellana y Sala, 2005).

Las gestiones y acciones que el sistema de educación superior ha llevado a cabo para el cumplimiento y fortalecimiento de los principios de equidad e igualdad de oportunidades, constituye un aspecto relevante que ha agilizado de manera significativa la práctica inclusiva universitaria; en respuesta al derecho a la educación. Para Chiroleu (2013) la expansión y cobertura sustentada en la aplicación de políticas inclusivas genera oportunidades sobre a aquellos grupos que requieren medidas complementarias y en esa misma línea agrega:

El ámbito educativo, supone el derecho al aprendizaje por parte de todos, independientemente de sus características individuales, con el fin de proporcionar atención al conjunto de demandantes según sus propias necesidades, lo que implica velar y generar condiciones adecuadas para la obtención de resultados favorables. (p.4)

Asímismo, Howe (2011), Shelvin, Kenny y Neela (2004), (citado en Aquino, García e Izquierdo, 2012) señalan que el éxito académico en la educación superior permite que las y los estudiantes con discapacidad sean parte de la vida productiva. De esta manera, el presente estudio evidenció cómo la propuesta educativa en la Sede Rodrigo Facio, abarca diferentes dimensiones de la accesibilidad en la vida universitaria estudiantil.

En cuanto a la accesibilidad de los diferentes escenarios del proceso formativo, las y los estudiantes encuestados hicieron señalamientos sobre diversidad de procesos, de esta manera, cuando el estudiantado se refiere al uso de los laboratorios (científicos, idiomas, computación) expresaron: "Algunos ni siquiera admiten estudiantes de la Facultad como el de Educación carecen de equipo como lectores de pantalla y magnificadores, los programas utilizados en los cursos de idioma no son accesibles para personas con discapacidad visual y los programas que utilizamos" (Encuesta) y "los laboratorios científicos pueden presentar problemas de accesibilidad pero no es un dato del que pueda dar fe. En cuanto a los demás laboratorios, la estructura y antigüedad de los edificios puede complicar su accesibilidad" (Encuesta). 
Al respecto, Chiny et al., (2008) comentan la importancia de brindar los apoyos necesarios que le permitan al estudiantado alcanzar un proceso educativo de calidad. En esa misma línea conceptual, Zubillaga del Río (2010) señala que la accesibilidad implica aspectos relacionados con el espacio físico, la formación académica y el aspecto social, con una perspectiva clara de las barreras que el entorno presenta para el acceso a la educación superior.

En este sentido el Reglamento de Régimen Académico Estudiantil, en el Artículo 3, inciso v, establece el principio de igualdad de oportunidades, como el reconocimiento de las necesidades educativas de los estudiantes y el empleo de recursos que garantice la oportunidad de acceso, en circunstancias equivalentes en la educación universitaria. Además, conceptualiza las adecuaciones como apoyos que se brindan según la necesidad educativa de la población estudiantil adscrita al Artículo 37 de este Reglamento. Con respecto a esta información se encontró que las adecuaciones recibidas por la mayor parte del grupo de estudiantes participantes en la investigación son:

- tiempo adicional

- grabación de clases,

- contar con material digital

- presentaciones en Power Point y videos antes de la clase,

- descripción de presentaciones en Power Point, videos o películas

- evaluación oral

- ubicación en el aula

- evaluación en tractos

- adecuación en el tamaño y tipo de letra

Por otra parte, en menor cantidad las y los estudiantes encuestados requieren iluminación en el aula, comunicación oral a corta distancia, apoyo en la toma de apuntes, evaluaciones grabadas, uso del sistema Braille en las evaluaciones, intérpretes de LESCO (Lengua de Señas Costarricense), digitalización de material, apoyos de software específico, apoyo de equipo tecnológico, tutor y facilitador. De estos datos se puede inferir que dichos apoyos responden a necesidades particulares de funcionalidad y condición del estudiante. Con lo cual se evidencia cómo la universidad responde ante la diversidad de necesidades educativas del estudiantado de manera consecuente con las políticas y normativa 
institucionales, contribuyendo al desarrollo de este en un entorno solidario y respetuoso de la diversidad humana (Stupp, 2005).

En relación con otros aspectos de la accesibilidad al proceso educativo, las y los participantes destacan que un elemento positivo es la realización de los equipos de apoyo que se hacen con la coordinación del Centro de Asesoría y Servicios a Estudiantes con Discapacidad (CASED). Por otra parte, las personas informantes son enfáticas al expresar la necesidad de generar y sistematizar mayores esfuerzos en el tema de las capacitaciones a las y los docentes. Además, insisten en realizar mayor divulgación hacia la comunidad universitaria, consecuentes con el fortalecimiento de los procesos de accesibilidad que la potencializan en procura del desarrollo de la población estudiantil y su participación en la sociedad. Al respecto Molina (2010) señala:

Respecto a programas y servicios que propendan por la inclusión es importante reconocer que las instituciones, como lo han sugerido autores como Naicker y García (1998), Arnaiz (2002), Verdugo (2003) y Díaz (2003), deben propender porque sus estudiantes no sólo reciban apoyos sino que participen activamente en las decisiones, soluciones, alternativas y políticas que les puedan generar bienestar en su vida universitaria. (p. 14)

Así, en cuanto al profesorado, el estudiantado participante en la investigación percibe grandes dificultades para el acceso al proceso educativo, manifiestan carencias en los siguientes aspectos:

- El profesorado no tiene mucha información sobre estrategias de accesibilidad.

- Falta de información sobre servicios de apoyo a los estudiantes.

- Poca disposición para adaptar las clases que son meramente visual.

- Excesivo uso del recurso visual en clases.

- Presentaciones con animación o con cuadros que limitan el acceso, principalmente aquellos estudiantes con discapacidad visual.

- Poca preparación del material de curso que no se puede dar en forma previa.

- Las evaluaciones no siempre responden a las necesidades del estudiante.

- Exámenes a libro abierto: esta modalidad limita al estudiante con discapacidad visual. 
Todos estas apreciaciones evidencian las fallas de un proceso educativo que incide en la formación profesional del estudiantado, de igual forma, señalan que aun cuando se cuenta con una fundamentación legal en materia de accesibilidad, respaldada por las políticas institucionales en materia de discapacidad y en el Reglamento de Régimen Académico Estudiantil, de la Universidad de Costa Rica, todavía se requiere de mayor esfuerzo institucional. En ese sentido, este estudio evidenció mediante información dada por el estudiantado, que el elemento actitudinal del profesorado, tiene que ir paralelo al conocimiento y dominio de estrategias y prácticas inclusivas como parte de la vivencia diaria en la universidad.

Por otra parte, la muestra participante opina sobre las carencias relacionadas con la vinculación directa al trabajo de aula, principalmente en la docencia universitaria, así sobre el profesorado señalan:

- No están fijos en la universidad lo que limita la consulta de estudiantes.

- No dan horas de consulta o que no se les asigna oficina para atender a los estudiantes.

- No atienden las necesidades del estudiante, hay falta de respeto.

- Profesores que no muestran interés o preocupación hacia la aplicación de las adecuaciones.

Además, se pronuncia sobre el elemento actitudinal expresando:

- Actitud negativa del profesorado.

- Docentes que no llegan al equipo de apoyo y no se informan.

- Profesorado que se les olvida el tipo de adecuación y su aplicación.

- Docentes que veces no aplican la adecuación del tiempo adicional.

- Falta de concientización sobre discapacidad y necesidades educativas.

En esta misma línea, Arguedas (2004) explica que el proceso de aplicación de las adecuaciones y la aceptación de la diversidad en el medio universitario, puede ser una fuente de aprendizaje y enriquecimiento para la comunidad universitaria. Además, rescata la trascendencia de profundizar más sobre las necesidades de ambas partes: profesorado y estudiantado, con lo cual se pueden generar mayores logros en la calidad de la formación, en procura de un proceso educativo accesible. 
Entre las aportaciones brindadas por las y los estudiantes de la muestra se dan comentarios críticos sobre la accesibilidad en la universidad e indican:

- Algunas y algunos profesores se complican con las adecuaciones.

- Los trabajos en grupos genera complicación en el desarrollo de los contenidos.

- Algunas y algunos profesores no toman en cuenta el estudiante y lo indispone.

- Algunas y algunos profesores se dirigen al facilitador no al estudiante con necesidades educativas (en casos de la o el estudiante sordo).

- Profesorado que no es educador.

Por otra parte, entre sus reflexiones el estudiantado participante en la investigación, también manifiestan algunos aspectos positivos en cuanto a la disposición del profesorado y apoyos en el tema de la aplicación de las adecuaciones:

- Algunos cursos se complica por los contenidos, pero siempre se encuentran soluciones.

- La disposición positiva de algunos profesores.

- Apoyos del personal de los Centros de Asesoría y Servicios Estudiantiles (CASE) y del Centro de Asesoría a Estudiantes con Discapacidad (CASED).

- Algunas unidades académicas dan mucho seguimiento a lo de las adecuaciones (Orientación y Educación Especial).

- Algunas y algunos docentes se esfuerzan para aplicar adecuaciones.

- Acercamiento del Profesor Consejero.

- Realización de Equipos de Apoyo.

Finalmente, las estudiantes y los estudiantes participantes resaltan la importancia de brindar capacitación al personal docente respecto a las adecuaciones y apoyos en el proceso educativo, principalmente en cuanto a las estrategias de aula.

De esta forma, hay una coincidencia de opiniones relacionadas a la priorización de capacitación sistemática al profesorado en la práctica universitaria, con el fin de adquirir mayores destrezas docentes hacia la diversidad del alumnado. En ese sentido, Sánchez (2011), destaca que el personal docente universitario no se siente con la preparación necesaria, para responder a los requerimientos de las y los estudiantes con discapacidad.

Al respecto, la UNESCO (2008) indica que es importante procurar un sistema educativo universitario que promueva un entorno de aprendizaje con la preparación 
necesaria para dar respuesta a la diversidad estudiantil, lo cual no solo se debe concebir a partir de las políticas y legislación existente, sino también desde la experiencia y vivencia estudiantil.

En cuanto a las adecuaciones recibidas por parte de la población estudiantil participante de la encuesta, un alto porcentaje indica que reciben tiempo adicional. Le siguen en proporción de requerimiento la adecuación de tener el material digital, presentaciones en Power Point y videos antes de la clase. Otras adecuaciones recibidas en menor cantidad son: ubicación en el aula, evaluación en tractos y requerimientos en cuanto al tamaño tipo de letra. $Y$ en porcentajes mínimos requieren iluminación en el aula, que el docente le hable de frente, apoyo en la toma de apuntes, evaluaciones orales, sistema Braille en las evaluaciones e intérpretes de LESCO. Así, en cuanto a las adecuaciones recibidas se observa, en el Gráfico 1, diversidad de opciones y respuestas para ser efectivo el acceso a la propuesta educativa universitaria.

\section{Gráfico 1}

Adecuaciones recibidas

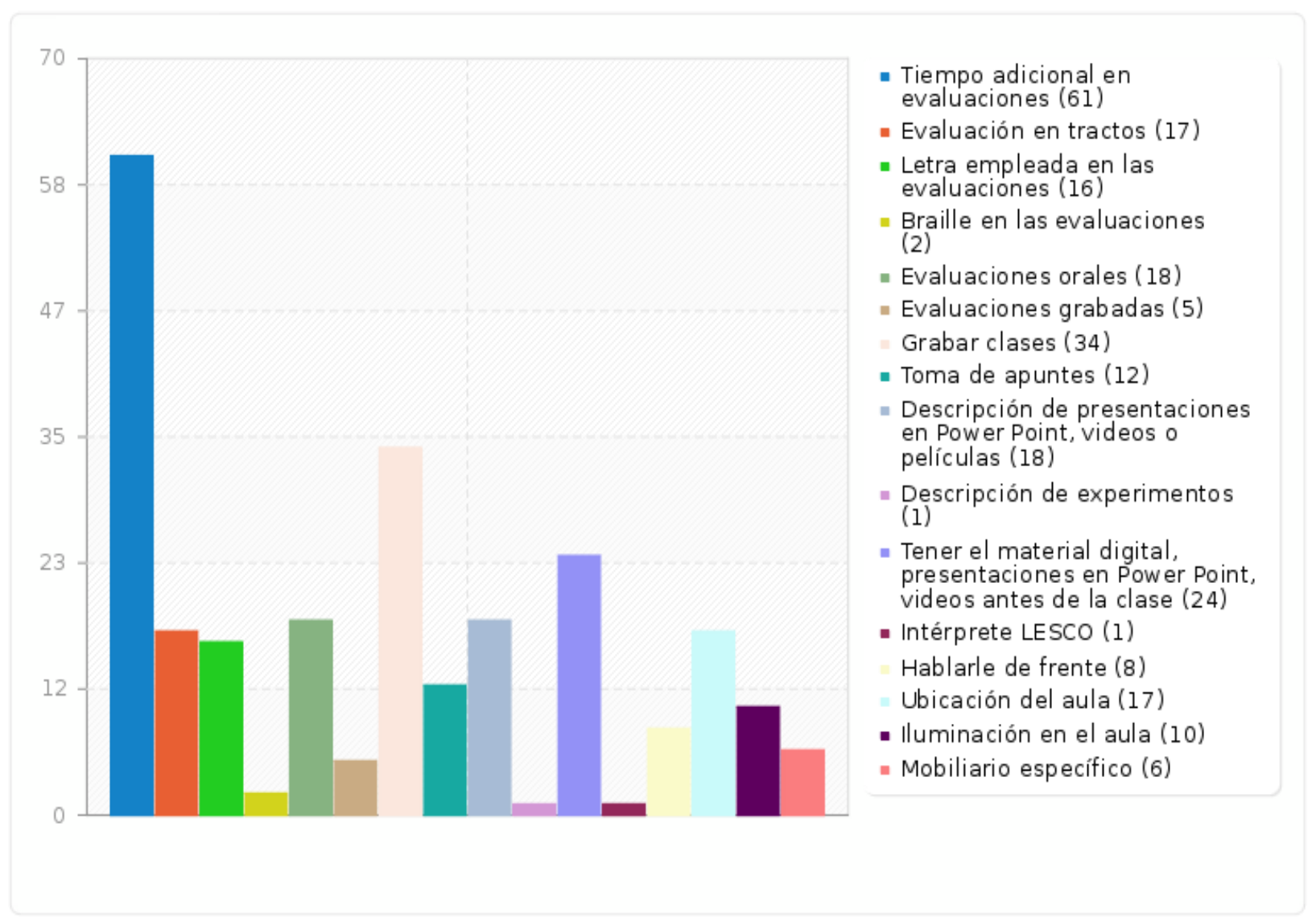

Fuente: Elaboración propia 
Con respecto a la información obtenida en el proceso investigativo sobre la dimensión de accesibilidad al proceso educativo, se encuentran requerimientos específicos según la condición del estudiante, la cual se conceptualiza a partir de una contextualización propia de la Universidad de Costa Rica, relacionada con las exigencias propias de las actividades de aprendizaje y evaluativos de los cursos matriculados, de esta manera se obtiene una variedad de respuestas acorde a situaciones particulares entrelazadas con la propuesta curricular que permite comprender de una forma integral la accesibilidad universitaria en todas sus dimensiones. Así se identificaron los siguientes apoyos:

- Estudiantes con condición emocional solicitan: tiempo adicional en evaluaciones, reprogramación de evaluaciones y trabajos, evaluaciones en recinto aparte, uso de más de un cuaderno de examen, no realizarle preguntas orales durante la clase, asistir a horas de consulta en forma individual, evaluaciones en tractos y apoyo de tutor.

- Estudiantes con discapacidad visual solicitan: tiempo adicional en evaluaciones, evaluaciones digitales, en tractos, con letra ampliada, tipo de letra específico, en sistema Braille, orales y en casa, además, textos a doble espacio, demostración directa al estudiante de movimientos corporales o gestos por parte docente, una prueba por día, uso de lupa, uso de marcador azul o negro, descanso en pruebas, aviso cambio mobiliario y descripción de experimentos.

- Estudiantes con discapacidad motora requieren: tiempo adicional en evaluaciones, evaluación en tractos, orales, descansos, marcar directamente en exámenes, evaluación en la tarde, reprogramación de evaluaciones, cambios posturales, disculpar llegadas tardías por desplazamiento interno y adaptación de ejercicios físicos.

- Estudiantes con condición auditiva requieren: tiempo adicional en evaluaciones, hablarle de frente, establecer el orden en actividades con rotación transcripción de grabaciones e Intérprete de LESCO

- Estudiantado con condición múltiple requieren apoyos según las necesidades particulares y funcionalidad, considerando las condiciones y áreas de discapacidad involucradas.

- Las y los estudiantes con problemas de aprendizaje solicitan las siguientes adecuaciones: tiempo adicional en evaluaciones y no tomar errores ortográficos como parte de la calificación final.

- Por otro lado, el estudiantado con déficit atencional solicita: tiempo adicional en evaluaciones y reprogramación de evaluaciones. 
- Finalmente, las y los estudiantes con diferentes situaciones de salud indican que requieren: tiempo adicional en evaluaciones, evaluación en tractos, grabar las clases, reprogramación de evaluaciones y trabajos, establecer orden en actividades de rotación, exámenes para resolver en la casa, descansos, letra ampliada en pizarra, uso de lupa, requerimientos en cuanto a tipo de letra, en crisis contactar persona clave, ingerir alimentos en clase, adaptación ejercicios físicos y apoyo de un tutor.

\section{Conclusiones y recomendaciones}

En relación con la accesibilidad al proceso educativo, la información obtenida a través de esta investigación ha logrado poner de manifiesto, desde la perspectiva del estudiantado, los logros y carencias que existen en materia de accesibilidad en el ámbito educativo de la Sede Rodrigo Facio. Los aportes de la población participante puntualizaron aspectos vinculantes relacionados con el derecho a la educación y el compromiso institucional, ante los desafíos que esta población enfrenta en el día a día del quehacer de la vida estudiantil.

Así, con la idea de que cada miembro de la comunidad universitaria es un elemento clave en el desarrollo de una universidad accesible, este estudio ofrece aportes hacia la comprensión de los aspectos que fortalecen la dinámica estudiantil universitaria, contextualizada mediante la participación de todos los elementos que conforman la vida estudiantil, lo cual a su vez sustenta acciones institucionales encaminadas a fortalecer el derecho a una educación pertinente y con excelencia.

De igual forma, este estudio refleja una realidad universitaria cambiante, sujeta a transformaciones constantes, tanto en el sector docente, en la población estudiantil y en el área administrativa, vinculados todos con el acceso al proceso educativo. Todo esto como producto de la apertura institucional para encontrar mayores y mejores respuestas a la oferta educativa universitaria, con una visión de la legitimización de los derechos de las poblaciones estudiantiles en condición de vulnerabilidad, tomar en cuenta la participación de ésta se considera un aspecto clave para el logro de una universidad accesible para todas y todos.

Por otra parte, se sugiere que por medio de las instancias formadoras de docentes, como son el Departamento de Didáctica Universitaria (DEDUN) y las Unidades Académicas, se valore la importancia de dar respuesta a los apoyos requeridos por esta población, según los ajustes razonables, la normativa institucional y la legislación nacional e internacional. De 
tal forma, que se valore la diversidad estudiantil como un componente intrínseco en una docencia inclusiva.

De igual forma, se propone fortalecer el proceso de educación permanente a la comunidad docente universitaria, sobre las estrategias didácticas e información general sobre los requerimientos de la población adscrita al artículo 37 del Reglamento de Régimen Académico Estudiantil, retomando como eje transversal las opiniones y sugerencias dadas por el estudiantado en cuanto a la relación docente-estudiante.

Finalmente, cabe resaltar que como producto de la investigación se construyó una Guía de Comprobación de Accesibilidad con el objetivo fundamental de responder a los requerimientos de la población estudiantil con necesidades educativas asociadas o no a discapacidad. Esta se planteó con la intención de ofrecer orientación sobre la aplicación de buenas prácticas inclusivas contemplando la accesibilidad desde la diversidad en el contexto universitario. El impacto que se espera que tenga esta Guía es que se constituya en un recurso que dinamice el proceso inclusivo y apoye en el fortalecimiento de las acciones que garanticen la igualdad de oportunidades, equidad y accesibilidad en el ámbito universitario.

\section{Referencias}

Asamblea Legislativa de la República de Costa Rica. (1996). Ley 7600 Igualdad de oportunidades para las personas con discapacidad. San José, Costa Rica: Editorama.

Asamblea Legislativa de la República de Costa Rica. (2008). Ley 8661 Convención sobre los derechos de las personas con discapacidad. San José, Costa Rica: COINDIS.

Arnáiz, Pilar. (2003). Educación Inclusiva, una escuela para todos. Málaga: Archidona Aljibe

Aquino, Silvia, García, Verónica e Izquierdo, Jesús. (2012). La inclusión educativa de ciegos y baja visión en el nivel superior. Un estudio de caso. Sinéctica, (39). Recuperado de http://sinectica.iteso.mx/?seccion=articulo\&lang=es\&id=552 la inclusion educativa de ciegos y baja vision en el nivel superior un estudio de caso

Arguedas, Irma. (2004). Reacciones de profesoras y profesores de la Universidad de Costa Rica ante la flexibilización del currículum para estudiantes con necesidades educativas especiales. Revista Actualidades Investigativas en Educación, 4(2). Recuperado de http://revista.inie.ucr.ac.cr/index.php/aie/article/view/57

Castellana, Montserrat y Sala, Ingrid. (2005). La universidad ante la diversidad en el aula. Revista Aula Abierta, (85), 57-83. Recuperado de http://www.researchgate.net/publication/28159835 La universidad ante la diversidad en el aula 
Chiny, Jeannette, Salas, Kattia y Vargas, Marie Claire.(2008). Accesibilidad para ingresar a la educación superior: desafíos y logros desde el enfoque de la diversidad. Revista Educare, 12(1). Recuperado de http://www.redalyc.org/pdf/1941/194114582011.pdf

Chiroleu, Adriana. (2013). ¿Ampliación de las oportunidades en la educación superior en la educación superior o democratización? Cuatro experiencias en América Latina. Revista Actualidades Investigativas en Educación, 13(3), 1-24. Recuperado de http://revista.inie.ucr.ac.cr/index.php/aie/article/view/584

Espinoza, Carlos, Gómez, Víctor y Cañedo, Carlos. (2012). El Acceso y la Retención en la Educación Superior de Estudiantes con Discapacidad en Ecuador. Revista Formación Universitaria, 5(6), 27-38. Recuperado de http://www.scielo.cl/pdf/formuniv/v5n6/art04.pdf

Gross, Martha y Stiller, Laura. (2014). Accesibilidad en la permanencia de estudiantes con necesidades educativas asociadas o no a discapacidad: Sede Rodrigo Facio (20122014) (Informe de Investigación). San José, Costa Rica: Instituto de Investigación en Educación. Universidad de Costa Rica.

Molina, Rocío. (2010). Educación superior para estudiantes con discapacidad. Revista de investigación, (70) Recuperado de http://www.urosario.edu.co/urosario files/aa/aa7a656b-30b9-4dec-87dd$\underline{6 a 174 a 507 c b a . p d f}$

Ramírez, Marcela. (2010). Las dimensiones de accesibilidad en la Universidad de Costa Rica, Sede Rodrigo Facio, un acercamiento desde las perspectivas de discapacidad y género. (Tesis para optar por el grado de maestría en Estudios Interdisciplinarios sobre Discapacidad). Universidad de Costa Rica, San José, Costa Rica.

Sánchez, Antonio. (2011). La Universidad de Almería ante la integración educativa y social de los estudiantes con discapacidad. Ideas y actitudes del personal docente e investigador. Revista de Educación, (354) 321-322. Recuperado de http://www.revistaeducacion.educacion.es/re354/re354 23.pdf

Sánchez, Antonio y Pulido Moyano, Rafael. (2007). El centro educativo: una organización de y para la diversidad. Granada: Grupo Editorial Universitario.

Salinas, Marcela, Lissi, María Rosa, Medrano, Daniela, Zuzulich, María Soledad, Hojas, AnaMaría, (2013). La inclusión en la educación superior: desde la voz de estudiantes chilenos con discapacidad. Revista Ibero-Americana de Educação, (63), 77-98. Recuperado de http://www.rieoei.org/rie63a05.pdf

Stupp, Roxana. (2005). Integración de Personas con Discapacidad a las Instituciones de Educación Superior en Costa Rica. Costa Rica: UNESCO.

UNESCO. (2008). Conclusiones y recomendaciones emanadas de la $48^{a}$ reunión de la Conferencia Internacional de Educación. Ginebra, Suiza: UNESCO_OIE. Recuperado de http://ibe.unesco.org/es/cie/48a-reunión-2008.html 
Zubillaga del Río, Ainara. (2010). La accesibilidad como elemento del proceso educativo: Análisis del modelo de accesibilidad de la Universidad Complutense de Madrid para atender las necesidades educativas de los estudiantes con discapacidad. (Memoria al grado de doctor). Universidad Complutense de Madrid, Departamento de Didáctica y Organización Escolar, Madrid, España. Recuperado de http://eprints.ucm.es/11430/1/T32369.pdf 